\title{
Estrategias para Mejorar la Sobrevida del Prematuro Extremo
}

\author{
MARÍA EUGENIA HÜBNER G. ${ }^{1}$, JULIO NAZER H. ${ }^{2}$, GUIDO JUÁREZ DE LEÓN \\ 1. Neonatología, Profesora Asistente, Hospital Clínico Universidad de Chile. \\ 2. Neonatología, Profesor Titular. Hospital Clínico Universidad de Chile. \\ 3. Obstetricia y Ginecología, Profesor Asistente Hospital Clínico Universidad de Chile.
}

\begin{abstract}
Strategies to Improve Survival in Extreme Premature Birth

Introduction: Biomedical advances have not decreased the number of premature deliveries. Newborns under 32 weeks constitute the most significant portion of infant mortality in developed countries. Objective: a literature review that identifies risk factors associated to premature labor and highlight strategies that increase survival. Results: Prenatal factor associated to early delivery include Black descent, maternal age, history of premature delivery, socioeconomic stratus, multiple deliveries and infections. Mortality is increased with earlier gestational age and lower fetal weight. Factors associated to increased survival include area of residence and place of birth, uterine transfer, variable levels of neonatal care, use of prenatal corticosteroids to accelerate pulmonary maturation, proactive management during childbirth, use of artificial surfactant and avoid use of steroids after delivery. Neonatal networks improve survival by potentiating best practices. Conclusions: Survival among these patients is enhanced by identification of risk factors for early delivery, knowing strategies that improve survival and planning for delivery in sites where best practices are offered.
\end{abstract}

(Key words: preterm birth, neonatal mortality, neonatal survival, neonatal intensive care, very low birth weight infant).

Rev Chil Pediatr 2009; 80 (6): 551-559

\section{RESUMEN}

Introducción: La ciencia biomédica no ha logrado disminuir la incidencia de partos prematuros. Los RN menores de 32 semanas, constituyen el principal determinante de la mortalidad infantil en los países más desarrollados. Los objetivos de esta revisión son identificar a través de la literatura médica los factores de riesgo asociados a parto prematuro y destacar las estrategias desarrolladas para mejorar la sobrevida de este grupo etario. Resultados: Entre los factores prenatales asociados a prematurez destaca la raza negra,

Trabajo recibido el 28 de octubre de 2008, devuelto para corregir el 16 de febrero de 2009, segunda versión el 02 de abril de 2009, aceptado para publicación el 01 de junio de 2009.

Correspondencia a:

Dra. María Eugenia Hübner G.

E-mail: mehubner@gmail.com 
la edad materna, historia de parto prematuro previo, el nivel socioeconómico, embarazos múltiples e infecciones. Constituyen factores de riesgo asociados a mortalidad neonatal, tener menor edad gestacional y peso, ser PEG. Dentro de las estrategias para mejorar la sobrevida destaca la regionalización, el traslado in útero, establecer diferentes niveles de atención en el cuidado neonatal, el uso corticoides pre natal para acelerar la madurez pulmonar, tomar una conducta proactiva al nacer, el uso de surfactante artificial y evitar tratamientos con corticoides después de nacer. Las redes neonatales permiten mejorar la sobrevida potenciando las mejores prácticas médicas. El lugar en que se nace es importante en la sobrevida del recién nacido, Conclusión: Identificar los factores de riesgo de parto prematuro y conocer las estrategias que mejoran la sobrevida de los RN menores de 32 semanas permite planificar el lugar de nacimiento y ofrecer las mejores prácticas clínicas destinadas a reducir la mortalidad de este grupo.

(Palabras clave: Parto prematuro, prematuro extremo, mortalidad neonatal, sobrevida neonatal, UTI neonatal, recien nacidos de muy bajo peso).

Rev Chil Pediatr 2009; 80 (6): 551-559

\section{Introducción}

La Organización Mundial de la Salud (OMS) define prematurez como aquel nacimiento de más de 20 semanas y menos 37 semanas de gestación. La Academia Americana de Pediatría (AAP) propone el límite de prematurez como aquel recién nacido $(\mathrm{RN})$ menor de 38 semanas, por el mayor riesgo de morbilidad que se describe entre las 37 y 38 semanas $^{1}$.

El término bajo peso se refiere a los $\mathrm{RN}$ menores de $2500 \mathrm{~g}$ independiente de la edad gestacional y no necesariamente prematuro.

En Europa la incidencia de los partos de pretérmino son alrededor del 5 al $7 \%$ de los nacidos vivos. En los EEUU alcanzan al 12\% ${ }^{2}$.

El parto pretérmino es el principal determinante de la mortalidad infantil en los países más desarrollados. Son los recién nacidos de muy bajo peso de nacimiento o menores de $1500 \mathrm{~g}$ (o menor de 32 semanas), los que constituyen el grupo de mayor riesgo. Este grupo de niños según diferentes publicaciones, comprenden alrededor del 1\% al 2\% de los nacidos vivos. En EEUU es 1,93\% $\%^{2}$. En Europa se describe tasas tan bajas como 0,04 en Luxemburgo y la más alta de 1,22 en UK y en España ${ }^{3}$. El proyecto MOSAIC que incluye diez países europeos informa una incidencia promedio de $0,9 \%$ con un rango de 0,76 a $1,3 \% \%^{3}$.

El Servicio de Salud de Chile en su informe técnico del año 2008, da una incidencia del $0,99 \%$, de recién nacidos menores de 32 semanas ${ }^{4}$.
En nuestro país, la mortalidad infantil ha declinado en forma vertiginosa desde tasas de 337 por mil en el año 1900, hasta 7,9 en el año $2008^{4-6}$. Los recién nacidos prematuros de muy bajo peso son responsable aproximadamente de 25 a $30 \%$ de la mortalidad infantil y del 50 a $70 \%$ de la mortalidad neonatal.

\section{Objetivos}

Ante la dificultad de prevenir el parto pretérmino, el objetivo de esta revisión es identificar, a través de lo publicado en la literatura médica, los factores de riesgo asociados a prematurez extrema y destacar como a lo largo del tiempo se han ido desarrollando estrategias destinadas a disminuir la mortalidad de los RN menores de $1500 \mathrm{~g}$. Se revisa lo publicado en la literatura inglesa e hispana a traves de Pubmed, Medline, base Cochrane y Lilacs entre los años 1995 a 2009.

\section{Factores de riesgos prenatales asociados a prematurez}

Una de las principales metas de las investigaciones biomédicas perinatales, ha sido la mejor comprensión de la etiopatogenia del parto prematuro y la identificación de los factores de riesgo que lo pueden desencadenar. Es así como se ha descrito los siguientes factores (tabla 1):

La raza, en United Kingdom y aún más en los EEUU, la incidencia de parto pretérmino en la población negra es más alta que en las muje- 
Tabla 1. Factores de riesgo asociados a prematurez

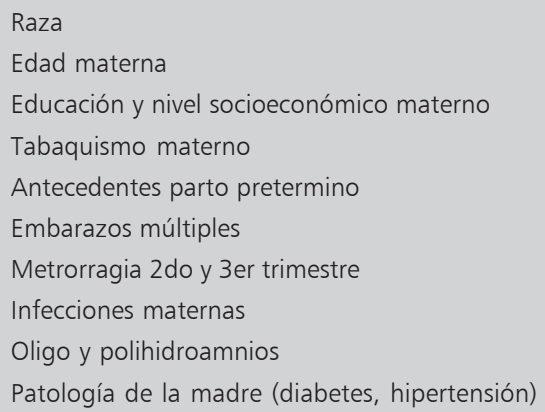

res blancas de la misma edad. Las mujeres de raza negra tienen una tasa de prematuridad del 16 al 18\%, comparado con el 7 al $9 \%$ en mujeres blancas. La razón para esta diferencia no es clara. Hay estudios recientes que asocian la raza negra paterna a prematurez $z^{2,7,8}$.

La edad materna, las mujeres menores de 17 y mayores de 35 años, tienen mayor riesgo de presentar parto prematuro ${ }^{9-10}$.

Importante predictor del parto de pretérmino es también, la educación materna y nivel socioeconómico y están relacionados con el grado de educación alcanzado por la madre. La literatura ha reportado que, la historia de haber tenido una mujer un parto pretérmino previo, tiene 2,5 veces más riesgo que se repita esta situación en su próximo embarazo ${ }^{2,10,11}$. Cuanto más temprana es la edad gestacional en que se produjo el parto pretérmino anterior, mayor es el riesgo de un nuevo parto de pretérmino espontáneo y precoz ${ }^{2,10}$.

El tabaquismo, las mujeres fumadoras tienen 20 a 30\% más probabilidad de tener un parto de prematuro. Importante es, que existen evidencias que el dejar de fumar disminuye el riesgo ${ }^{2,9,11}$.

Los embarazos múltiples, casi el 50\% de los embarazos dobles y prácticamente todos los embarazos múltiples con más de dos fetos, terminan antes de completar las 37 semanas, siendo su duración promedio, más corta cuanto mayor es el número de fetos que crecen in útero en forma simultánea (36 semanas para los gemelares, 33 para los triples y 31 para los cuádruples $)^{11}$. La tasa de gemelos aumentó en un 55\% entre los años 1980 y 1998 en los
EEUU, debido al uso de drogas que estimulan la ovulación y el desarrollo de técnicas de fertilización in vitro y reproducción asistida. Esta tasa disminuyó cuando sólo se permitió el desarrollo de dos embriones en el útero.

La presencia de metrorragia, sea por desprendimiento de placenta marginal o por placenta previa, es un factor de riesgo tan importante como el embarazo múltiple.

Las infecciones maternas, la bacteriuria asintomática y otras infecciones sistémicas como neumonía bacteriana y pielonefritis pueden provocar contracciones uterinas y terminar en un parto prematuro $^{11}$. La enfermedad períodoncial también se encuentra asociada a este riesgo.

Otros factores que se han asociado con prematurez han sido, la presencia de oligo y polihidramnios, la cirugía abdominal materna en el segundo y tercer trimestre y patología materna como diabetes e hipertensión.

Los procesos fisiológicos mediante el o los cuales se desencadena el trabajo de parto todavía se desconocen, pero se han identificado algunos marcadores bioquímicos y anatómicos que podrían ayudar a predecir el trabajo de parto de pretérmino. Con una prueba de fibronectina fetal negativa, y una longitud cervical por ecografía de $30 \mathrm{~mm}$ o más, la probabilidad de tener un parto pretérmino es menor al $1 \%{ }^{11,12}$. Las numerosas prácticas empleadas para prevenir o tratar los síntomas de parto prematuro, como el reposo en cama, sedación, monitorización de la actividad uterina, el uso de betamiméticos orales o intravenosa, tratar las infecciones asintomáticas y la vaginosis, el uso de nutrición complementaria etc. no han mostrado suficientes evidencias acerca de su efectivi$\operatorname{dad}^{2,11}$.

Principales estrategias desarrolladas para mejorar la sobrevida de los prematuros extremos (tabla 2).

\section{Organización de la atención neonatal}

Frente a esta realidad la Neonatología ha debido desarrollar numerosas estrategias destinadas a mejorar la sobrevida de los recién nacidos prematuros. En 1970, en EEUU se inicia, la regionalización del cuidado neonatal que junto con favorecer una mejor atención se optimiza recursos y se establecen diferentes 
Tabla 2. Estrategias asociadas a mayor sobrevida del prematuro

\begin{tabular}{ll}
\hline $\begin{array}{l}\text { Organización de la } \\
\text { atención }\end{array}$ & $\begin{array}{l}\text { Manejo clínico } \\
\text { del RN }\end{array}$ \\
\hline $\begin{array}{l}\text { Regionalización cuidado } \\
\text { neonatal }\end{array}$ & Uso surfactante \\
Establecer niveles de atención & Corticoides prenatales \\
Transporte in útero & $\begin{array}{l}\text { Guías de reanimación } \\
\text { neonatal }\end{array}$ \\
Activo manejo obstétrico & $\begin{array}{l}\text { Limitar uso corticoides } \\
\text { post natal }\end{array}$ \\
Redes neonatales & Redes neonatales \\
\hline
\end{tabular}

niveles de cuidado, el nivel tres comprende atención en unidad de tratamiento intensivo (UTI) implementada con alta tecnología, que cuenta con la posibilidad de otorgar diferentes modalidades de ventilación mecánica, monitorización y cirugía neonatal ${ }^{13}$.

Entre 1970 y 1980, se organiza el transporte in útero de los embarazos de riesgo, y se define un activo cuidado obstétrico del RN inmaduro $^{13}$.

En 1986, en los EEUU no sólo comienza a aumentar las tasas de sobrevida de los prematuros sino que también la tasas de morbilidad, los días de hospitalización y los costos, siendo éstos excepcionalmente altos al compararlos con los RN de término, entonces surgen algunas interrogantes como: ¿que es ser demasiado pequeño?, dónde y cómo ponemos el límite?, ¿que es un cuidado apropiado?, ¿como disminuir la morbilidad?, para contestar estas preguntas y tomar conductas basadas en evidencias se crea NICHD (National Institute of Child Health and Human Development Neonatal Research Network $)^{13}$. De esta manera los centros de atención neonatal quedan en redes lo que permite unificar criterios, optimizar recursos, contener los costos, identificar prácticas médicas que logran mejores resultados y establecer normas de conducta.

\section{Desarrollo de estrategias en el manejo clínico de los $R N$}

Las principales innovaciones a partir de ese período incluyeron, la introducción del surfac- tante en 1990 y se establecen nuevas modalidades ventilatorias para tratar los problemas respiratorios de los prematuros.

El NICHD en 1994, difunde el consenso de usar corticoides pre natal en las embarazadas entre 24-34 semanas que presenten signos de amenaza de parto prematuro con la finalidad de hacer profilaxis de enfermedad hialina, logrando reducir la mortalidad, el síndrome de dificultad respiratoria y la hemorragia intracraneana en los prematuros ${ }^{15}$.

En 2001, nacen las guías de reanimación neonatal ampliamente difundidas ${ }^{16}$.

En el 2002, la Academia Americana de Pediatría a través del comité del feto y recién nacido establece el consenso de limitar el uso de corticoides post natal, por las complicaciones evidenciadas de su uso tales como perforación gastrointestinal, hipertensión, hiperglicemia, sepsis, crecimiento disminuido y alteraciones en el neurodesarrollo ${ }^{17}$.

\section{Factores de riesgo asociados a menor sobrevida del prematuro (tabla 3)}

Las publicaciones perinatales, son consistentes en describir, que dentro de los factores de riesgo la edad gestacional y el peso de nacimiento son determinantes de la sobrevida de los prematuros extremos, menor edad y menor peso, determina menor sobrevida.

Los porcentajes de mortalidad, son mayores para el sexo masculino que para el femenino, a similares rangos de edad gestacional y peso de nacimiento, principalmente en aquellos $\mathrm{RN}$ que están en el límite de la viabilidad ${ }^{18,19}$.

Los RN pequeños para la edad gestacional (PEG) tienen mayor riesgo de desarrollar síndrome de dificultad respiratoria y mayor morta-

Tabla 3. Factores de riesgo asociados a mortalidad del prematuro

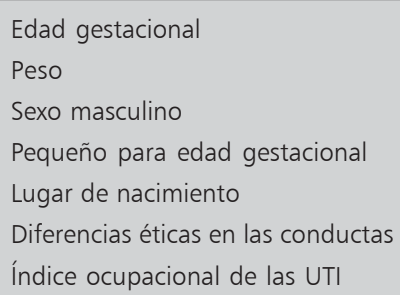


lidad en cada edad gestacional, siendo estadísticamente significativa, desde las 25 a las 29 semanas de gestación, en relación a los $\mathrm{RN}$ adecuados para la edad gestacional ${ }^{20}$.

\section{Resultados de algunas estrategias destinadas a mejorar la sobrevida}

La aparición de las Redes Colaborativas como la NICHD, the Vermont Oxford Network $(\mathrm{VON})^{21}$ Oxford, la red canadiense ${ }^{21}$, la red australiana $^{23}$, las europeas entre ellas EuroNeoStat $^{24}$ y los estudios multicéntricos han permitido una mejor organización del trabajo en equipo, identificar factores de riesgo de morbimortalidad, promover la cultura del cambio en el personal tanto médicos como enfermeras y personal que atiende a los recién nacidos y permitir modificar las prácticas clínicas, basados en evidencias científicas, contribuyendo así a aumentar la sobrevida de los prematuros extremos. Hay numerosas publicaciones que comparan diferentes estrategias que han permitido mejorar la sobrevida.

La NICHD ${ }^{13,25}$ publica sus resultados en tres períodos destacando la mejoría de la sobrevida especialmente en algunos grupos aplicando nuevas estrategias. Comprende todos los nacimientos de cada uno de los centros participantes, se considera tres épocas, 1987-1988 período pre surfactante, 1993-1994 post surfactante y moderado uso de corticoides prenatal y
1999-2000 post surfactante con un amplio uso de corticoides prenatal. El uso de corticoides prenatal aumentó de $16 \%$ en 1987 a $79 \%$ en 1999/2000. Con esto se logró una reducción importante del SDR de $67 \%$ a $42 \%$. En la tabla $4^{13}$, se puede observar la sobrevida por edad gestacional en los tres períodos descritos y vemos que la sobrevida para los $\mathrm{RN}$ a las 24 semanas en el período 1987-1988 fue de 34\% y aumenta a $59 \%$ en 1900-2000, para las 25 semanas sube de $54 \%$ en el primer período a $72 \%$ en el tercer período.

En la tabla 5, se muestra la sobrevida por peso de nacimiento, los RN de peso entre $501 \mathrm{y}$ $600 \mathrm{~g}$ aumentan la sobrevida de $21 \%$ en el primer período a $39 \%$ en el año 1999/2000, los RN con peso entre 701 y 800 g aumenta la sobrevida de $53 \%$ a $77 \%$ en el mismo período.

Entre el grupo de 1000 g-1 500 g la mortalidad cayó de $13 \%$ a $7 \%$ destacando una disminución del $43 \%$ entre el primer período y el segundo.

En Suecia, Hakansson ${ }^{26}$, analiza el beneficio de una conducta pro activa en Suecia del Norte que consiste en la centralización de la atención neonatal, uso de corticoides y tocolíticos pre natal, hacer una evaluación adecuada de la edad gestacional, cesárea y conducta activa al nacer versus conducta selectiva en Suecia del Sur. Los datos son obtenidos de un registro nacional de nacimientos del centro epidemio-

Tabla 4. Sobrevida por edad gestacional

\begin{tabular}{cccc}
\hline Edad gestacional (sem) & $\mathbf{1 9 8 7 - \mathbf { 1 9 8 8 }}$ & $\mathbf{1 9 9 3 - \mathbf { 1 9 9 4 }}$ & $\mathbf{1 9 9 9 - 2 0 0 0}$ \\
\hline$\leq 23$ & 23 & 24 & 30 \\
24 & 34 & 56 & 59 \\
25 & 54 & 70 & 72 \\
\hline
\end{tabular}

*Tomado de Fanaroff A Seminars in Perinatology 2003;27: 285 (13).

Tabla 5. Sobrevida para recién nacidos con peso nacimiento 501 - 800 g

\begin{tabular}{cccc}
\hline Peso nacimiento $(\mathbf{g})$ & $\mathbf{1 9 8 7} \mathbf{- 1 9 8 8}$ & $\mathbf{1 9 9 3 - \mathbf { 1 9 9 4 }}$ & $\mathbf{1 9 9 9 - 2 0 0 0}$ \\
\hline $501-600$ & 21 & 30 & 39 \\
$601-700$ & 33 & 56 & 59 \\
$701-800$ & 53 & 72 & 77
\end{tabular}

*Tomado de Fanaroff A Seminars in Perinatology 2003; 27: 285 (13). 
lógico. Se define sobrevida como estar vivo al un año de edad. Se estudia el período de tiempo comprendido 1985-1999. No hay diferencias en las características epidemiológicas de los RN estudiados. La incidencia de menores de $1500 \mathrm{~g}$ en el Norte fue de 1,08 y en el sur 0,85. Entre los RN menores de $800 \mathrm{~g}$ la sobrevida fue de $52 \%$ en el Norte y $41 \%$ en el Sur. Entre las $22-$ 25 semanas de gestación hubo un $51 \%$ de sobrevida en el Norte versus $37 \%$ en el Sur. Entre los años 1995 y 1999 la sobrevida para los RN del Norte fue 2,5 veces mejor que la de los niños nacidos en el Sur.

E1 85\% de los RN menores de 25 semanas nacidos en el Norte lo hicieron en hospital tipo terciario, los del Sur sólo el 56\%.

La región del Norte es más grande y la población está muy esparcida, por lo tanto, la transferencia en útero no depende primariamente de la proximidad a un nivel III sino que de una actitud activa y de una expedita organización del transporte. En dicha región hubo aumento de la sobrevida, menor número de RN con Apgar bajo, menor número de RN fallecidos dentro de las 24 horas de vida.

Stoelhorst $\mathrm{t}^{27}$, compara dos cohortes que formaron parte de un proyecto sobre prematurez, LFUPP 1996/1997 que incluyó RN menores de 32 semanas semanas y el POPS-1983 un estudio de la era pre-surfactante, también RN menores de 32 semanas y/o menores de $1500 \mathrm{~g}$. La estrategia realizada para mejorar la atención fueron aumentar la centralización del cuidado perinatal y usar corticoides pre natal. El porcentaje de transporte extrauterino disminuye de $61 \%$ el año 1983 a $35 \%$ el año 19961997. El uso de corticoides pre natal aumenta de 6 a $73 \%$ en el mismo período. Se observó una disminución de la mortalidad de los menores de 27 semanas de $76 \%$ a 33\%. En grupo total de prematuros la mortalidad disminuyó del $30 \%$ a $11 \%$ Lorenz $^{28}$, compara diferentes estrategias en el manejo del prematuro extremo en New Jersey (NJ) y Netherland (NETH) en dos poblaciones contemporáneas de recién nacidos entre 23 y 26 semanas. El monitoreo fetal electrónico, la operación cesárea y la ventilación asistida fueron más comunes en $\mathrm{NJ}$ en la que se observó una mortalidad a los 28 días de $45,9 \%$ versus $73,2 \%$ en NETH lugar en que además ningún niño menor de 25 semana sobrevivió.

\section{Otros factores de riesgo asociados a mortalidad}

Se observa en los estudios realizados que: 1) el lugar de nacimiento constituye un factor importante en el pronóstico del prematuro dado por las diferencias en tecnología, en las prácticas obstétricas y neonatales y en la proporción de enfermeras especializadas en la atención neonatal ${ }^{3,29,30}$.

Johansson en Karolinska describe una mortalidad para los recién nacidos entre 23 y 27 semanas ajustada por riesgo de dos veces mayor en un hospital general versus un hospital universitario ${ }^{30}$.

2) La diferencias éticas en la conducta a seguir frente al nacimiento de un $\mathrm{RN}$ prematuro extremo incide en la morbimortalidad, por ejemplo en Noruega la alta mortalidad bajo las 28 semanas de gestación refleja el consenso de no reanimar activamente al nacer a estos niños ${ }^{3}$.

En una publicación reciente se concluye que la mortalidad en los RN de muy bajo peso nacimiento es más baja en los hospitales con UTI neonatal que tienen nivel tres de atención y con alto volumen de pacientes ${ }^{31}$. Sin embargo, se ha descrito también que un 3 ) índice ocupacional de la unidad de cuidados intensivos neonatales sobre el $80 \%$ aumenta la morbilidad $^{32}$.

\section{Prematurez en Chile}

Al analizar la situación de los prematuros en Chile observamos que la mortalidad neonatal en 1914 era de 115 por 1000 recién nacidos vivos, el año 2003 es de 5 por 1000 , ocurriendo el $75 \%$ de las muertes en la primera semana de vida o mortalidad neonatal precoz. Los principales factores determinantes en esta impactante disminución ha sido la mejoría socioeconómica del país, el control del embarazo, la atención profesional del parto, la regionalización de la atención perinatal ${ }^{4-33}$.

En Chile, el inicio del cuidado intensivo neonatal tiene lugar el año 1980 con la implementación de las UTI neonatales a lo largo del país. En 1992 el curso de reanimación neonatal 
para neonatólogos de Santiago y regiones. En 1994 nace el programa de atención de los RN con cardiopatías congénitas, en 1998 se comienza a generalizar el uso de surfactante artificial $^{33}$.

Las redes neonatales que han contribuido a la mejoría de la sobrevida también existen en nuestro país. En enero de 2000 se inicia el seguimiento en red de prematuros en todos los servicios públicos de salud del país, (representa el $75 \%$ de los RN) se crea una base de datos, en la que, se ingresan los RN menores de $1500 \mathrm{~g}$ y/o menores de 32 semanas, cuenta con 28 unidades de cuidados intensivos neonatales y 35 policlínicos de seguimiento ${ }^{4}$. En el informe del quinquenio 2000-2004 se observa un aumento de la sobrevida de 70 a $76 \%$, especialmente importante es la mejoría en el grupo que pesa $750 \mathrm{~g}$ a $999 \mathrm{~g}$ que aumenta la sobrevida de 58 a $70 \%{ }^{4}$.

Chile participa también en red en el grupo Colaborativo NEOCOSUR que reúne a recién nacidos menores de $1500 \mathrm{~g}$ provenientes de 16 centros de 5 países: Argentina, Perú, Paraguay y Uruguay, que en su publicación en el año 2000 encontró una sobrevida del grupo global de $73 \%{ }^{34}$. Se destaca en este estudio una gran variabilidad entre los diferentes centros, que va desde una sobrevida global para los menores de $1500 \mathrm{~g}$ de $89 \%$, comparable con los mejores centros de países desarrollados, a un 50\% en el centro de peor resultado. En el año 2006 NEOCOSUR ${ }^{35}$ publica un aumento en el uso de profilaxis de enfermedad de membrana hialina con corticoides prenatal, mayor uso de surfactante artificial y soporte ventilatorio, destacando una menor morbilidad con disminución de las sepsis precoz y de la hemorragia intracraneala.

\section{Comentario}

A lo largo del tiempo se han ido desarrollando numerosas estrategias que han mostrado tener impacto sobre la morbi-mortalidad del prematuro extremo, tales como, la organización del cuidado perinatal, la regionalización, el lugar de nacimiento, el contar con UTI neonatal, la conducta proactiva, las redes neonatales. Con este continuo progreso del cuidado perinatal y con una conducta activa en el momento de nacer el límite de la viabilidad se ha trasladado hacia edades gestacionales menores permitiendo que RN de 22 a 24 semanas sobrevivan. Hay un acuerdo internacional de que no se espera sobrevida en menores de 22 semanas $^{36}$.

Los RN menores de 24 semanas, tienen alta posibilidad de fallecer durante el primer año de vida y los que sobreviven aproximadamente la mitad de ellos tienen alteraciones de diferente grado en el neurodesarrollo.

El cuidado de esto RN implica decisiones éticas, sociales, médicas y económicas complejas.

Es probable que no cambie el límite de la viabilidad, se ha mejorado la sobrevida, sin embargo, debe progresar también la calidad del cuidado perinatal de manera de mejorar el pronóstico de los RN prematuros extremos. El cuidado de estos RN niños y futuros adultos afecta a todas las áreas y disciplinas de la medicina.

Hacia el futuro se espera un mejor conocimiento de los factores genéticos que predisponen al parto prematuro y el desarrollo de estrategias que permitan su prevención, mientras no se logre esto la Neonatología debe seguir avanzando de manera de mejorar el pronóstico de estos $\mathrm{RN}$ y permitir que todos reciban una atención en el nivel más alto de competencia.

\section{Referencias}

1.- American Academy of Pediatrics. Comitte on fetus and newborn: Levels of neonatal care. Pediatrics 2004; 114: 1341-7.

2.- Tucker J, Mc Guire $W$ : Epidemiology of preterm infant BJM 2004; 329: 675-8.

3.- Zeitlin J, Draper E, Kollée L, et al: Differences in rate and short term outcome of live birth before 32 weeks of gestation in Europe in 2003: results from MOSAIC cohort. Pediatrics 2008; 121; e936-44.

4.- Morgues $M$, Reyes $C$ : Informe técnico. Recién nacidos con menos de 32 semanas de edad gestacional. Sistema Nacional de Servicios de Salud de Chile. Quinquenio 2000-2004.www.prematuros.cl 2008.

5.- INE 2004.

6.- Aguila A: Indicadores neonatales. En Nazer J. Ramírez R. ed Neonatología. Hospital Clínico Universidad de 
Chile 2001; 5-8. www.hospital.uchile.cl/paginas/ revistahospital/NeonatologiaWeb

7.- Adams MM, Elam-Evans LD, Wilson HG, Gilberto $D A$ : Rates of and factors associated with rcurrence of preterm delivery. JAMA 2000; 283: 1591-6.

8.- Simham HN, Krohn MA: Paternal race and preterm infant. Am J Obstet Gynecol 2008; 198: 644.e1644.e6.

9.- Robert TH: Prevention of premature birth: do pediatricians have a rol. Hall R Pediatrics 2000; 105; 1137 40.

10.- Goldenberg RL: The management of preterm labor. Obstet Gynecol 2002; 100: 1020-37.

11.- Bhutta Z, Darmstadt G, Hasan B, Haws R: Community-based interventions for improving perinatal and neonatal health outcomes in developing countries: a review of the evidence. Pediatrics 2005; 115: 519-617.

12.- Welsh A, Nicolaides $K$ : Cervical screening for preterm delivery. Curr Opin Obstet Gynecol 2002; 14 (2): 195 202.

13.- Fanaroff A, Hack M, Walsh M: The NICHD neonatal research network: changes in practice and outcomes during the first 15 years. Seminars in Perinatology 2003; 27: 281-7.

14.- Blackmon L: The rol of the hospital of birth on survival of extremly low-birthweight, extremely preterm infants. Neorewiews 2003; 147e-152.

15.- Bethesda MD: Report of the consensus development conference on the effect of corticosteroids for the fetal maduration on perinatal outcomes. National Institutes of Health. November 1994 (NIH publication N 95-3784).

16.- International guidelines for neonatal resuscitation: An expert from the guidelines 2000 for cardiopulmonary resuscitation and emergency cardiovascular care: international consensus of science. Pediatrics 2000; 106: 1-16.

17.- American Academy of Pediatrics Committee on Fetus and Newborn: Postnatal corticoesteroids to treat or prevent chronic lung disease in preterm infants. Pediatrics 2002; 109: 330-8.

18.- Mc Donald H: Perinatal care at the Threshold of viability. Pediatrics 2002: 110: 1024-7.

19.- Hübner ME, Ramírez R: Sobrevida, Viabilidad y Pronóstico del Prematuro. Rev Méd Chile 2002; 130: 931-8.

20.- Regev R, Reichman B: Prematurity and intrauterine growth retardation-double jeopardy? Clin Perinatol 2004; 31: 453-73.
21.-(VON) Ohlinger $J$, Brown $M$, Laudert $S$, et al: Development of potentially better practices for the neonatal intensive care unit as a culture of collaboration: Cominication, accountability, respect, and emporwerment. Pediatrics 2003; 111: e475-81.

22.- http://www.canadianneonatalnetwork.org/index. shtml2008

23.- http://www.psn.org.au/index.php?option=com_content \&task=view\&id=57\&Itemid=84.2008

24.- Valles I Soles M, Pejoain J, Paillás Alonso CR: EuroNeoStat Un sistema europeo de información sobre los resultados de la asistencia a recién nacidos de muy bajo peso. An Pediatr 2006; 65: 1-4.

25.- Horbar J, Rogowski J, Plsek P, et al: Collaborative Quality Improvement for Neonatal intensive care. Pediatrics 2001; 107: 14-22.

26.- Hakansson S, Farooqi A, Holmgren P, Serenius F, Högberg $U$ : Proactive management promotes outcome in extremely preterm infants; a population-based comparison of two perinatal management strategies. Pediatrics 2004; 114: 58-64.

27.- Stoelhorst G, Rijken M, Shirley E, et al: Changes in neonatology: comparision of two cohorts of very preterm infants (gestational age $<32$ weeks): the Project on Preterm and Small for Gestational Age Infants 1983 and the Leiden Follow-up Project on Prematurity 1996-1997. Pediatrics 2005; 115: 396-405.

28.- Lorenz J, Paneth N, Jetton R, Ouden L, Tyson J: Comparison of managent strategies for extreme prematurity in New jersey and the Netherland: outcome and resource expanditure. Pediatrics 2001; 108: 1269-74.

29.- Hamilton $K$, Redshaw M, Tarnow-Mordi $W$ : Nurse staffing in relation to risk-adjusted mortality in neonatal care. Arch Dis Child Fetal and Neonatal Ed 2007; 92: F99-F103.

30.- Johansson S, Montgomery M, Ekbom A, et al: Preterm delivery,level of care,and infant death in Sweden: a population-based study. Pediatrics 2004; 113 : 1230-5.

31.- Phibbs C, Baker L, Caughey A, Danielsen B, Schmitt $S$ : Level and volume of neonatal intensive care and mortality in very -low-birth.weight infants. N England Med 2007; 356: 2165-75.

32.- The UK Neonatal Staffing Study Group: Patient volume, staffing, and workload in relation to riskadjusted outcomes in a random stratified simple of UK neonatal intensive care units: a prospective evaluation. Lancet 2002; 359: 99-107.

33.- González R, Merialdi M, Lincetto $O$, et al: Reduction 
in neonatal mortality in Chile between 1990 and 2000 Pediatrics 2006; 117: 949-54.

34.- Grupo Colaborativo Neocosur: Very Low Birh Weigt infants outcome in 11 Southamerican NICU'S. J Perinatol; 2002: 202-7.

35.- Tapia JL, D Apremont I, Quezada M, Marshall G,
Gederlini A: Neocosur informe annual. Rev Hosp Mat Ramón Sardá 2007; 26: 116-31.

36.- Pignotti MS, Donzelli G: Perinatal care al the Threshold of viability: an international comparison of practical guidelines for the treatment of extremely preterm birth. Pediatrics 2008; 121: e93-e98. 